Artículo

\title{
Interacción genotipo-ambiente en el rendimiento de genotipos de papa
}

\author{
Víctor Vásquez Arce ${ }^{1}$ \\ Pablo Huerta Fernández ${ }^{2 \S}$ \\ Héctor Cabrera Hoyos ${ }^{3}$ \\ Luis Jiménez Díaz
}

${ }^{1}$ Universidad Nacional de Cajamarca. Jirón Los Fresnos núm. 239, Urb. El Ingenio, Cajamarca, Perú. Tel. 976301328. (vvarce@ hotmail.com). ${ }^{2}$ Universidad Nacional Agraria La Molina. Calle Madrid núm. 116, Depto. 101, Miraflores, Lima, Perú. Tel. 971129290. CP. 18. (apablohuerta@gmail.com). ${ }^{3}$ Estación Experimental Baños del Inca-INIA. Pasaje libertad 331, Barrio San Sebastián, Cajamarca, Perú. Tel. 974160067. (hach8@ hotmail.com). ${ }^{4}$ Universidad Nacional Agraria La Molina. Pasaje Monteagudo núm. 154, Breña, Lima, Perú. Tel. 947019953. CP. 140104. (jdl@lamolina.edu.pe).

${ }^{\S}$ Autor para correspondencia: apablohuerta@gmail.com.

\section{Resumen}

Se evaluaron ocho genotipos resultantes de cinco ciclos de selección recurrente de Solanum tuberosum, utilizando el diseño bloques completos al azar con tres repeticiones. Hay diferencias altamente significativas en el peso total y tubérculos totales de los diferentes genotipos en seis ambientes. El análisis combinado detectó diferencias significativas para ambientes, genotipos e IGE. Los efectos localidades, genotipos, genotipos x localidad y genotipos x ambiente fueron altamente significativos. Los mejores índices ambientales para peso de tubérculos y tubérculos totales se lograron en las localidades de: Santa Clotilde, La Púcara, Marcobamba y Santa Margarita. El genotipo 399062.115 fue considerado como el genotipo estable, consistente y con alto potencial de rendimiento $46.01 \mathrm{t} \mathrm{ha}^{-1}$. Los genotipos: 396012.266 y 393377.159 con rendimientos de $42.01 \mathrm{t} \mathrm{ha}^{-1}$ y $38.66 \mathrm{t} \mathrm{ha}^{-1}$, respondieron mejor a condiciones desfavorables y consistentes.

Palabras clave: Solanum tuberosum L., Andina, producción.

Recibido: enero de 2021

Aceptado: marzo de 2021 


\section{Introducción}

Un programa de mejoramiento genético de papa (Solanum tuberosum L.) se dirigirá al potencial de rendimiento, a la estabilidad y resistencia a Phytophthora infestans. El potencial de rendimiento considerado como una variable cuantitativa es un proceso fisiológico muy complejo que está determinado por el genotipo, el ambiente y la interacción genotipo x ambiente (Vásquez 1988; Pérez et al., 2010. La estabilidad se refiere a la capacidad que tiene la planta para expresar su potencial de rendimiento en una amplia gama de ambientes (Vásquez 2013; Tirado et al., 2019) y la resistencia estará dirigida al tipo horizontal (Rubio et al., 2016). Un estudio sobre la interacción genotipo $\mathrm{x}$ ambiente (IGE) para rendimiento en papa mostró que todos los genotipos tuvieron una respuesta diferencial a los efectos ambientales (Vásquez et al., 2019; Tirado et al., 2018).

En la Región Cajamarca muchos genotipos (clones) son sembrados en diferentes condiciones ambientales, habiéndose observado efectos muy marcados del ambiente sobre la expresión fenotípica de ellos, por lo que la variabilidad genotípica que se aprecia es debido a la variación genética más el efecto del medio ambiente (IGE), por tanto debe trabajarse para disminuir el efecto de la IGE; a través, del uso de diseños experimentales adecuados y con un buen manejo agronómico oportuno (Vásquez et al., 2019).

Los objetivos de la presente investigación fueron: determinar la existencia o no de la interacción genotipo $\mathrm{x}$ ambiente (IGE) y su efecto en el rendimiento de peso de tubérculo y número total de tubérculos y detectar genotipos estables usando el modelo sugerido por Eberhart y Russell (1966).

\section{Materiales y métodos}

De los seis ambientes estudiados, tres experimentos se establecieron en la provincia de Cajamarca, dos en la provincia de Chota ambos pertenecientes a la Región de Cajamarca y un experimento en la provincia de Sánchez Carrión (Región La Libertad). Todos instalados bajo condiciones de secano (campaña 2016-2017). Las características de los seis sitios se anotan en el Cuadro 1.

Cuadro 1. Características geográficas, climáticas y edáficas de los cinco ambientes donde se realizó la evaluación de los diez genotipos de papa.

\begin{tabular}{|c|c|c|c|c|c|c|c|}
\hline & Localidad & Año & $\begin{array}{l}\text { Latitud } \\
\text { Longitud }\end{array}$ & $\begin{array}{l}\text { Altitud } \\
(\mathrm{m})\end{array}$ & $\begin{array}{c}\text { Temperatura } \\
\text { anual }\left({ }^{\circ} \mathrm{C}\right)\end{array}$ & $\begin{array}{l}\text { Precipitación } \\
\text { (mm) }\end{array}$ & Tipo de suelo \\
\hline \multirow[t]{4}{*}{ Cajamarca } & Santa Clotilde & 2016 & -7.1443438 & 2900 & 13.5 & 107 & Franco \\
\hline & & & -78.322611 & & & & arcilloso \\
\hline & Santa & 2016 & -7.114251 & 2950 & 13.2 & 110.5 & Franco \\
\hline & Margarita & & -78.3041 & & & & arenoso \\
\hline \multirow[t]{3}{*}{ Chota } & Chucmar & 2016 & $\begin{array}{l}-6.409631 \\
-78.577461\end{array}$ & 3200 & 12.1 & 106 & $\begin{array}{c}\text { Franco arcillo } \\
\text { arenoso }\end{array}$ \\
\hline & Marcobamba & 2016 & $\begin{array}{l}-14.05948 \\
-73.52772\end{array}$ & 3036 & 14.3 & 95.5 & $\begin{array}{l}\text { Franco } \\
\text { arcilloso }\end{array}$ \\
\hline & La Púcara & 2016 & $\begin{array}{l}-15.97993 \\
-71.45988\end{array}$ & 3600 & 10.2 & 115.6 & Franco \\
\hline $\begin{array}{l}\text { Sánchez } \\
\text { Carrión }\end{array}$ & Chugay & 2016 & $\begin{array}{l}-7.7825 \\
-77.8678\end{array}$ & 3378 & 10 & 132.8 & $\begin{array}{c}\text { Franco arcillo } \\
\text { arenoso }\end{array}$ \\
\hline
\end{tabular}

Servicio Nacional de Meteorología e Hidrología-SENAMHI (2017). 
Se consideraron ocho genotipos de papa de la Estación Experimental Agropecuaria Baños del IncaCajamarca (INIA). Los genotipos representan lo más avanzado de las cruzas de $S$. andigena x $S$. andígena y selección recurrente por cinco ciclos (B1C1 a C1B5) y la población resultante fue mejorada buscando resistencia a $P$. infestans, precocidad, adaptación, apariencia de tubérculos en formas más definidas, ojos superficiales y rendimientos de tubérculos comerciales superiores a sus parientes cercanos (Gastelo et al., 2010). Los genotipos evaluados se muestran en el Cuadro 2.

\section{Cuadro 2. Genotipos de papa evaluados en el experimento.}

\begin{tabular}{cccc}
\hline Núm. & Genotipos & Pedigree & Grupo \\
\hline 1 & 399075.26 & $(387348.20 \times 389746.2)$ & B3C1 \\
2 & 399076.12 & $(391004.10 \times 393280.58)$ & B3C1 \\
3 & 396012.266 & $(395285.5 \times 395282.3)$ & B3C2 \\
4 & 399062.115 & $(395274.1 \times 395319.2)$ & B1C5 \\
5 & 393377.159 & $(395285.5 \times 395282.3)$ & B1C5 \\
6 & 399058.12 & $(395266.2(B 1 C 4046.2) \times 395282.3(\mathrm{~B} 1 \mathrm{C} 406.2 .3))$ & B1C5 \\
7 & 393077.54 & & B1C5 \\
8 & 399062.108 &
\end{tabular}

Para la evaluación de los ocho genotipos se usó un diseño de bloques completos al azar con tres repeticiones por ambiente. La parcela experimental constó de dos surcos de tres metros de longitud separados a $30 \mathrm{~cm}$ entre golpe y a $1 \mathrm{~m}$ entre surco. Se colocó una semilla brotada por golpe. A la cosecha se evaluó el peso de tubérculos totales y número de tubérculos totales.

\section{Resultados y discusión}

En los análisis de varianza individuales se encontraron diferencias altamente significativas entre genotipos $(p<0.01)$ para rendimiento total y número de tubérculos totales en los seis ambientes (Santa Clotilde, Santa Margarita, Chucmar, Marcobamba, la Púcara y Chugay). La prueba de Bartlett indicó que las varianzas fueron homogéneas y los supuestos de normalidad de los errores se cumplieron (Vásquez, 2014). Los coeficientes de variación oscilaron entre $5.23 \%$ a 2.93\%, considerándose como buenos para darle precisión a los ensayos (Vásquez, 2013). Hubo significancia estadística $(p \leq 0.01)$ para localidades, genotipos e interacción genotipo $\mathrm{x}$ ambiente (IGA) (Cuadro 3). La heterogeneidad entre ambientes estuvo relacionada con diferencias en altitud, tipo de suelo, temperaturas y precipitación durante los años de estudio (Cuadro 1).

Las diferencias para genotipos evidenciaron la existencia de variabilidad genética en rendimiento de peso total y número de tubérculos totales. Estos resultados concuerdan con los de Tirado et al. (2018) evaluaron papas con pulpa pigmentada en cuatro ambientes obteniendo significancia estadística para ambientes, genotipos e IGE. La IGA demuestra que los genotipos de papa tienen un comportamiento diferente en los diversos ambientes probados. Estos resultados son concordantes con los de Vásquez et al. (2019), quienes al evaluar adaptación y rendimiento de seis clones en ocho ambientes encontraron diferencias para genotipos y para la interacción genotipo por medio ambiente. Salomón et al. (2015), probó trece progenies de papa en dos ambientes, encontraron significación estadística al $1 \%$ de probabilidades para progenies y para la IGA. 
La heterogeneidad entre ambientes estuvo relacionada principalmente con diferencias en altitud, tipo de suelo, temperaturas y precipitación durante los años de estudio (Cuadro 1). Se observó que, la relación de los cuadrados medios de genotipos con la de los cuadrados medios de la IGA es 57.22, lo cual indica la mayor importancia de los genotipos sobre la interacción (Cuadro 3). Esto demuestra la existencia de la variabilidad genética, que nos permite la selección de genotipos superiores (Vásquez, 1988).

Cuadro 3. Análisis de varianza combinado (cuadrados medios) para peso total y número de tubérculos totales.

\begin{tabular}{cccc}
\hline \multirow{2}{*}{ Fuente de variación } & \multirow{2}{*}{ Grados de libertad } & \multicolumn{2}{c}{ Cuadrados medios } \\
\cline { 3 - 4 } & & Peso total & Tubérculos totales \\
\hline Localidad & 12 & $4.2669^{* *}$ & 173.7842 \\
Rep/Loc & 7 & 0.2132 & 1.4347 \\
Genotipos & 35 & $112.0806^{* *}$ & 27.3993 \\
Loc x genotipos & 84 & $1.4734^{* *}$ & 13.8199 \\
Error & 143 & 0.4168 & 3.556 \\
Total & & & \\
\hline
\end{tabular}

$\mathrm{R}^{2}=0.9608,0.8392 ; \mathrm{CV}(\%)=3.47,12.18 \quad \overline{\mathrm{y}} .=37.11,12.63$.

De acuerdo, al Cuadro 3 el medio ambiente representó $2.38 \%$ de la variación del rendimiento total, el genotipo $87.65 \%$ y la interacción $\mathrm{G} \times \mathrm{E}$ de $5.76 \%$. Sobre la base de la contribución relativa de suma de cuadrados, los genotipos tienen una mayor contribución, seguida de la interacción GxE y por último la contribución de los ambientes. Para el número total de tubérculos el ambiente representa $46.78 \%$, los genotipos, refiere 10.31 y $25.99 \%$ de la variación representa a la IGA. Estos resultados difieren con lo mencionado por Tirado et al. (2018), Salomón et al. (2015) quienes hallaron que la mayor contribución de la IGE fue de 60.82 y $70.13 \%$, la del genotipo de $26.03 \%$ y $10.3 \%$.

El Cuadro 4, muestra que el mejor ambiente para la evaluación de los genotipos fue Chugay, Santa Clotilde y la Púcara con promedios de 38.66, 37.16 y $37.1 \mathrm{t} \mathrm{ha}^{-1}$ respectivamente, superando estadísticamente a las cinco localidades restantes, así como al promedio de la región $14 \mathrm{t} \mathrm{ha}^{-1}$ (MINAGRI 2017). No se encontró diferencias estadísticas entre las localidades de Marcobamba, Chucmar y Santa Margarita, cuyos rendimientos oscilaron entre $36.98 \mathrm{t} \mathrm{ha}^{-1}$ hasta $36.24 \mathrm{t} \mathrm{ha}^{-1}$ respectivamente.

Cuadro 4. Prueba de comparación múltiple (Duncan) de las medias para rendimiento (t ha-1) de los genotipos en estudio por localidad sobre el índice ambiental (Ij).

\begin{tabular}{|c|c|c|c|c|c|}
\hline \multirow{2}{*}{ Clave } & \multirow{2}{*}{ Localidad } & \multicolumn{2}{|r|}{ Peso total } & \multicolumn{2}{|c|}{ Núm. total de tubérculos } \\
\hline & & $\left(\mathrm{t} \mathrm{ha}^{-1}\right)$ & Índice ambiental (Ij) & Núm. promedio & Índice ambiental (Ij) \\
\hline 6 & Chugay & $38.66 \mathrm{a}$ & 1.57 & $11.5388 \mathrm{~d}$ & -5.65 \\
\hline 1 & Santa Clotilde & $37.16 \mathrm{~b}$ & 0.01 & $17.3575 \mathrm{ab}$ & 2.8 \\
\hline 5 & La Púcara & $37.1 \mathrm{~b}$ & 0.01 & $13.8965 \mathrm{c}$ & -3.31 \\
\hline 4 & Marcobamba & $36.98 \mathrm{bc}$ & -0.12 & $17.0406 \mathrm{~b}$ & 2.2 \\
\hline 3 & Chucmar & $36.5 \mathrm{bc}$ & -0.59 & $14.2927 \mathrm{c}$ & -1.55 \\
\hline 2 & Santa Margarita & $36.24 \mathrm{c}$ & -0.86 & $18.7654 \mathrm{a}$ & 5.5 \\
\hline
\end{tabular}


El modelo de parámetros de estabilidad identificó como los mejores ambientes para rendimiento a la localidad de Chugay, Santa Clotilde y la Pucara, por los valores de sus índices ambientales (Ij) positivos; es decir, superiores a la media general (37.11 t ha $\mathrm{ha}^{-1}$ y a los ambientes con mal comportamiento (Marcobamba, Chucmar y Santa Margarita), por tener índices ambientales negativos; es decir, menores a la media general. Para el número de tubérculos totales los mejores ambientes fueron Santa Clotilde, Marcobamba y Santa Margarita por tener sus índices ambientales positivos. Estos resultados son superiores a los obtenidos por Gastelo et al. (2010) quienes, al evaluar dichos genotipos en cuatro localidades de Puno, obtuvieron rendimientos promedios que oscilaron entre 18 a $28 \mathrm{t} \mathrm{ha}^{-1}$.

\section{Parámetros de estabilidad}

Se encontró significación al 1\% de probabilidades para genotipos y G x Amb (lineal) para peso de tubérculos y número total de tubérculos (Cuadro 5). No se encontró significación para la desviación conjunta para las dos variables en los ocho genotipos. Las diferencias entre genotipos permiten detectar aquellos con mejor potencial genético. La significancia de la interacción genotipo x medio ambiente (lineal), revela que existe una respuesta diferencial cambiante de los genotipos a través de ambientes para las variables respuesta estudiadas. Esto nos propone que es posible seleccionar genotipos con mejor potencial genético y estabilidad en una serie de ambientes como lo señalan Eberhart y Russell (1966). Cabe mencionar que, en el rendimiento promedio de los 8 genotipos, se observó variación en el potencial productivo. Este resultado considera la importancia de conducir experimentos en tiempo y espacio, para observar la repuesta de las variedades y genotipos en diferentes condiciones de prueba (Becker y León, 1988; Vásquez, 1988; Fikere et al., 2008).

Cuadro 5. Análisis de la varianza (cuadrados medios) para la estabilidad según Eberhart y Russell (1966), de ocho genotipos de papa evaluados en seis localidades para peso y número totales de tubérculos.

\begin{tabular}{cccc}
\hline \multirow{2}{*}{ Fuente de variación } & \multicolumn{3}{c}{ Cuadrados medios } \\
\cline { 2 - 4 } & Grados de libertad & Peso de tubérculos & Núm. total de tubérculos \\
\hline Total & 143 & & \\
Genotipos & 7 & $53.74^{* *}$ & $9806.95^{* *} \mathrm{M} 3$ \\
Amb + (G x Amb) & 40 & 0.87 & 7321.4 \\
Amb (lineal) & 1 & 10.24 & 292961.236 \\
G x Amb (lineal) & 7 & $10.24^{* *} \mathrm{M} 2$ & $46.825^{* *} \mathrm{M} 2$ \\
Desviación conjunta & 32 & $0.6562 \mathrm{M} 1$ & $4948.3324 \mathrm{M} 1$ \\
399075.26 & 4 & $1.1453^{*}$ & $3441.8992 \mathrm{~ns}$ \\
399076.12 & 4 & 0.2877 & $1294.6063 \mathrm{~ns}$ \\
396012.266 & 4 & 0.2336 & $15885.5058 \mathrm{~ns}$ \\
399062.115 & 4 & 0.1436 & $886.6341 \mathrm{~ns}$ \\
393377.159 & 4 & $1.3125^{* *}$ & $9256.964 \mathrm{~ns}$ \\
399058.12 & 4 & 1.0756 & $1706.4922 \mathrm{~ns}$ \\
393077.54 & 4 & 0.7024 & $1348.9553 \mathrm{~ns}$ \\
399062.108 & 4 & 0.3746 & $5749.1537 \mathrm{~ns}$ \\
Error combinado & 84 & 0.4168 & 3.4815 \\
\hline
\end{tabular}




\section{Interpretación de los parámetros de estabilidad}

En la Cuadro 6, se presenta la clasificación de los genotipos de papa evaluados, considerando primeramente los criterios utilizados por Eberhart y Russell (1966), en donde se emplearon el comportamiento promedio del genotipo a través de ambientes, su coeficiente de regresión y la desviación de la regresión.

Cuadro 6. Rendimiento de peso total y número de tubérculos de ocho genotipos de papa, incluyendo los parámetros de estabilidad.

\begin{tabular}{|c|c|c|c|c|c|c|c|}
\hline \multirow{2}{*}{ Núm. } & \multirow{2}{*}{ Genotipos } & \multicolumn{3}{|c|}{ Peso total } & \multicolumn{3}{|c|}{ Número total de tubérculos } \\
\hline & & $\left(\mathrm{t} \mathrm{ha}^{-1}\right)^{+}$ & bi & $S_{\mathrm{dj}}^{2}$ & Tubérculos totales ${ }^{+}$ & bi & $\mathrm{S}_{\mathrm{dj}}^{2}$ \\
\hline 1 & 399075.26 & $35.26 \mathrm{e}$ & $1.076^{* *}$ & 1.001 & $16.77 \mathrm{~b}$ & 1.2741 & 9.7718 \\
\hline 2 & 399076.12 & $37.34 \mathrm{~d}$ & 0.065 & 0.149 & $11.09 \mathrm{~b}$ & 0.9165 & 3.0505 \\
\hline 3 & 396012.266 & $38.63 \mathrm{c}$ & 1.01 & 0.095 & $16.77 \mathrm{a}$ & 0.6261 & 38.1428 \\
\hline 4 & 399062.115 & $46 \mathrm{a}$ & $1.103^{* *}$ & 0.005 & $11.45 \mathrm{a}$ & 1.0665 & 0.0892 \\
\hline 5 & 393377.159 & $42.01 \mathrm{~b}$ & 1.415 & 1.174 & $11.39 \mathrm{~b}$ & 0.6578 & 20.7872 \\
\hline 6 & 399058.12 & $32.3 \mathrm{~g}$ & $0.617^{* *}$ & 0.937 & $14.23 \mathrm{ab}$ & 1.2633 & 3.7924 \\
\hline 7 & 393077.54 & $33.8 \mathrm{f}$ & 2.194 & 0.563 & $12.06 \mathrm{~b}$ & 0.8699 & 1.6213 \\
\hline 8 & 399062.108 & $31.43 \mathrm{~h}$ & 0.582 & 0.236 & $12.89 \mathrm{~b}$ & 1.2258 & 11.3696 \\
\hline
\end{tabular}

${ }^{+}=$medias con la misma letra son estadísticamente iguales (Duncan, 0.05 ) $; \bar{y}_{.}=37.11 \mathrm{t} \mathrm{ha}^{-1} ; \bar{y}_{.}=12.63$.

Para identificar y clasificar a los genotipos de papa por su estabilidad, con base en los estimadores de los coeficientes de regresión (bi) y desviaciones de regresión $\left(\mathrm{S}_{\mathrm{di}}{ }^{2}\right)$ para las seis categorías propuestas por Carballo y Márquez (1970), se consideraron los siguientes escenarios con relación a los valores de los coeficientes de regresión $\mathrm{bi}=1, \mathrm{bi}<1, \mathrm{bi}>1$ y las desviaciones de regresión $\mathrm{S}_{\mathrm{di}}^{2}=0, \mathrm{~S}_{\mathrm{di}}^{2}>0$, de estas se identificaron tres de los ocho posibles grupos (Cuadro 6), de la forma siguiente: 1) los genotipos 396012.266 (3) y 399062.115 (4) presentaron bi= 1 ; y $\mathrm{S}_{\text {dii }}^{2}$ ) 0; es decir, son considerados como genotipos estables (Eberhart and Russell, 1966; Carballo y Márquez, 1970), cuyos rendimientos superan al rendimiento promedio $\left(37.11 \mathrm{t} \mathrm{ha}^{-1}\right) ; 2$ ) coeficientes de regresión bi< 1 y desviaciones de regresión $S_{\text {di }}^{2}=0$, el genotipo 399076.12 (2) responde con buena respuesta en ambientes desfavorables consistentes; y 3) el genotipo 393377.159 (5) presenta coeficientes de regresión bi> 1 y desviaciones de regresión $\mathrm{S}_{\mathrm{di}}^{2}>0$, es un genotipo con buena respuesta en ambientes favorables pero inconsistente.

En lo referente al número total de tubérculos (Cuadro 6) se identificaron dos grupos de los ocho genotipos evaluados: 1) los genotipos 399075.26 (1), 399062.115 (4), 399058.12 (6) y 399062.108 (8) presentaron coeficientes de regresión bi> 1 y desviaciones de regresión $\mathrm{S}_{\mathrm{di}}^{2}>0$ son de buena respuesta en ambientes favorables pero inconsistentes; y 2) los genotipos 399076.12 (2), 396012.266 (3), 393377.159 (5) y 393077.54 (7) con bi< 1 y la $\mathrm{S}^{2}{ }_{\mathrm{di}}>0$, son aquellos genotipos con buena respuesta en ambientes desfavorables e inconsistentes.

Estos resultados son concordantes con los reportes de Pérez et al. (2007); Vásquez et al. (2019), quienes encontraron valores que caracterizan a genotipos con respuesta mejor en ambientes buenos, en condiciones desfavorables y consistentes, así como inconsistentes. 
Para el número total de tubérculos, según el modelo de Eberhart y Russell (1966), los mejores ambientes para los genotipos en estudio fueron Santa Margarita, Santa Clotilde y Marcobamba, con 5.5, 2.8 y 2.2 tubérculos, por tener valores de los índices ambientales (Ij) positivos.

\section{Conclusiones}

Las diferencias altamente significativas que se observaron entre genotipos, entre localidades y en su interacción (IGA) sugieren que hay suficiente variabilidad genética para seleccionar genotipos sobresalientes, que los ambientes de la región Cajamarca son heterogéneos y que la IGA significativa dificulta la identificación de genotipos estables.

Los mejores índices ambientales para peso de tubérculos y número de tubérculos totales fueron en Santa Clotilde, La Púcara, Marcobamba y Santa Margarita, ubicados en Cajamarca y Chota. El modelo de Ebarhart y Russell identificó a los genotipos: 399062.115 (4), 393377.159 (5) y 396012.266 (3). con $46.01 \mathrm{t} \mathrm{ha}^{-1}, 42.01 \mathrm{t} \mathrm{ha}^{-1}$ y $38.66 \mathrm{t} \mathrm{ha}^{-1}$ superiores al promedio $\left(37.11 \mathrm{tha}^{-1}\right) \mathrm{y}$ con $b i=1, s^{2} d i=0$, considerados como genotipos estables. El genotipo 399062.115 (4) para número total de tubérculos presentó un coeficiente de regresión bi= 1 y una desviación de regresión $\mathrm{s}^{2} \mathrm{di}=$ 0 considerado como genotipo estable.

\section{Literatura citada}

Becker, H. C. and León, J. 1988. Stability analysis in plan breeding. Plant Breed. Review. 101(1):1-23.

Carballo, C, A. y Márquez, S. 1970. Comparación de variedades de maíz del Bajío y la Mesa Central por su rendimiento y estabilidad. Agrociencia. 5(1):129-146.

Eberhart, S. A. and Russell, W. A. 1966. Stability parameters for comparing varieties. Crop Science. 6(1):36-40.

Fikere, M.; Tadesse, T. and Letta, T. 2008. Genotype - environment interactions and stability parameters for grain yield of faba bean (Vicia faba L.) genotypes grown in South Eastern Ethiopia. Int. J. Sustain. Crop Prod. 3(6):80-87.

Gastelo, M.; Arcos, J.; Landeo, J. y Bonierbale, M. 2010. Nuevas variedades de papa Solanum tubersoum spp. andígena (B1C5), obtenidas a través de la selección participativa por los agricultores de las comunidades del altiplano, Puno-Perú. In: I Congreso Internacional de Investigación y Desarrollo de papas nativas. Quito-Ecuador. 137-138 pp.

Márquez, Y.; Salomon, J. y Acosta, R. 2020. Análisis de la interacción genotipo ambiente en el cultivo de la papa (Solanum tuberosum L.). Cultivos Tropicales. 4(1):1-14.

MINAGRI. 2017. Características de la producción nacional y de la comercialización en Lima metropolitana. Boletín pruducción nacional de papa-Perú. 30 p.

Pérez, L.; Vásquez, G. y Sahagún, J. 2007. Estabilidad del rendimiento de genotipos de papa (Solanum tuberosum L.). Rev. Fitotec. Mex. 30(003):279-284.

Pérez, D.; Gonzáles, A. Huerta, M.; Rivera, A.; Sahagún, J.; Balbuena, A.; Rubí, A. y Gutiérrez, F. 2010. Variabilidad genética, diversidad fenotípica e identificación de genotipos sobresalientes de papa. Rev. Mex. Cienc. Agríc. 1(4):579-592.

Rubio, C. O. A.; Cadena, H. M. A. y Flores, L. R. 2016. Comparación de la resistencia horizontal y vertical contra el tizón tardío de la papa en Toluca. Rev. Mex. Cienc. Agríc. 7(4):755-766. 
Salomón, J.; Castillo, J.; Arzuaga, J. A.; Torres, W.; Caballero, A.; Varela, M. and Betancourt, V. M. H. 2015. Análisis de la interacción progenie-ambiente con minitubérculos a partir de semilla sexual de papa (Solanum tuberosum L.) en Cuba. Cultivos Tropicales. 36(2):83-89.

SENAMHI. 2017. Servicio Nacional de Meteorología e Hidrología. Registros meteorológicos. Lima, Perú.

Tirado, C.; Vásquez, V. y Narro, L. A. 2019. Estabilidad de rendimiento y adaptabilidad de híbridos de maíz tolerantes a suelos ácidos en base a las características del análisis GGE biplot. Aci. Avances. 11(17):50-63.

Tirado, R.; Tirado, L. y Mendoza, J. 2018. Interacción genotipo ambiente en rendimiento de papa (Solanum tuberosum L.) con pulpa pigmentada en Cutervo, Perú. Chilean J. Agric. Anim. Sci., ex Agro-Ciencia. 34(3):191-198.

Vásquez, V. 1988. Mejoramiento genético de la papa. ${ }^{\text {ra. }}$ (Ed.). Amaru editores. Consejo Nacional de Ciencia, Tecnología e Innovación Tecnológica-CONCYTEC. Lima, Perú. 149-153 pp.

Vásquez, V. 2013. Experimentación agrícola. $1^{\text {ra. }}$ (Ed.). Consejo Nacional de Ciencia, Tecnología e Innovación Tecnológica-CONCYTEC. Lima, Perú. 417-436 pp.

Vásquez, V. 2014. Diseños experimentales con SAS. 1 ${ }^{\text {ra. }}$ (Ed.). Consejo Nacional de Ciencia, Tecnología e Innovación Tecnológica-CONCYTEC, Fondo Nacional para el Desarrollo de la Ciencia, Tecnología e Innovación Tecnológica-FONDECYT. Lima, Perú. 206-210 pp.

Vásquez, V.; Cabrera, H.; Jiménez, D. y Colunche, A. 2019. Estabilidad del rendimiento de genotipos de papa (Solanum tuberosum L.). Ecología Aplicada. Dpto. Académico de Biología, Universidad Nacional Agraria La Molina. Lima-Perú. 18(1):59-65. 